\title{
Optimum Design of Anode for Dow Electrolytic Cell
}

\author{
Othman S. Hamad, Omed Gh. Abdullah, and Mudhaffer M. Ameen
}

\begin{abstract}
Numerical techniques was used to solve the Laplace's equation in Dow electrolytic cell, the computation of voltage distribution in anode was carried out by using the method of successive over relaxation finite difference method. The developed finite difference program is constructed to study the effect of various design parameters or operate change on the performance on the anode; performance is judged from the dissipated energy, life time of anode, quantity of magnesium produce. A novel anode design is suggested to minimize the dissipated energy increase the lifetime, and relativity large quantity of magnesium produce.
\end{abstract}

Index Terms-Dow cell, finite difference, dissipated energy, magnesium produce.

\section{INTRODUCTION}

The knowledge of the consumption energy in every part of the Dow cell is very important in both scientific and economical points of view. There are some physical phenomena which happen during the cell operation as a result of passing high electric current [1],[2], which is followed by many other physical occurrences like thermal effects. The electric and magnetic fields have direct effect on the cell efficiency, so the calculation and analysis of potential and electric field distribution represent an important stage in the design of anode in the Dow cell.

Thus comprehension should be taken into account to understand the physical phenomena for cell constituent, in order to obtain the best designs available for these constituent $[3,4]$. In view of amount of electric energy consumed in the production of pure $\mathrm{Mg}$ there is considerable incentive for improvement of the Dow cell.

The work discussed here begins after some difficulties process had been experienced with many of the anodes in the cells, after the commissioning of the plat it was found that the voltage drop across the anode was higher and more variable than had been expected. Although this high voltage drop means that increased ohmic heat generation could also impair the anode performance in the cell [5]. At the outset it was realized that the poor and variable characteristics of the cost iron joint were the principal causes of the trouble and it was decided therefore, to quantify the voltage distributions throughout the anode, using a mathematical model, as a function of the degree of electrical contact at the joint, as a result, a better understanding of the physics of the system was expected to emerge and form this it would be possible to make constructive criticism of the existing joint making

Manuscript received August 4, 2012; Revised September 23, 2012

O. S. Hamad and M. M. Ameen are with the College of Science-Education, University of Salahaddin-Erbil, Kurdistan Region-Iraq (e-mail: othmansalemk@yahoo.com).

O. G. Abdullah is with the Department, School of Science, Faculty of Science and Science Education, University of Sulaimani, P.O.Box: 342, Kurdistan Region-Iraq (e-mail: omed.abdullah@univsul.net). procedures and to recommend ways of improving them.

To quantify the voltage distributions the anode, which measured $(62 \times 46 \times 150) \mathrm{cm}$ high, was approximated to a uniform solid cylinder of equivalent base area. This enabled it to be simplified to a two dimensional system with cylindrical symmetry without it was felt, significantly the validity of the model.

Laplace's equation was used to define the steady state voltage distributions throughout the system with appropriate constraints placed on the equation of the boundaries. Actual values of carbon/cost iron contact resistance required to define conditions of this boundary, were measured in the laboratory.

Simulation were carried out to reproduce existing conditions on the plant and also to observe the benefits of modifying the joint design and taken different dimensions of anode and stub.

\section{MODEl CONSTRUCTION}

A half section of the simplified anode geometry is shown in Fig. 1. In the model the cavity was assumed to have a smooth vertical side. The carbon cast iron interface regions are shown along line $\mathrm{E}$ and $\mathrm{F}$, and carbon electrolyte interface region was postulated along line $\mathrm{D}$. current was assumed to flow normally across any interface area and its direction is shown by the arrows. For cavity the interface are shown to have a finite thickness, while in practice, it is infinitesimally thin. The dimensions of the anode were considered to be variables in the model so different geometry could be explored.

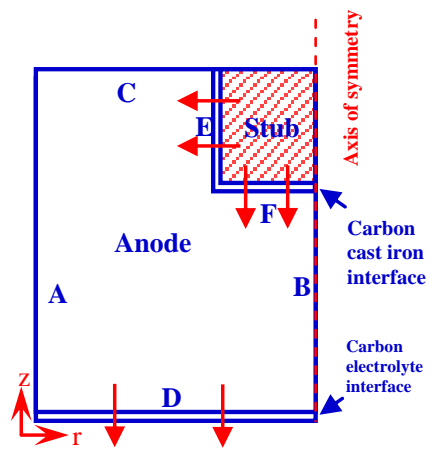

Fig. 1. Half section of simulated anode, arrows show current direction.

\section{BOUNDARY CONDITIONS}

Across the boundary line (A) no current was assumed to flow. Therefore, this line boundary separates the two Laplacean regions one infinitely permeable and flux crosses the boundary normally. So the finite differences equation corresponding to this line is:

$$
V_{i j}=\frac{1}{4}\left[2 V_{(i-1, j)}+V_{(i, j-1)}+V_{(i, j+1)}\right]
$$


Line boundary (B) represents the line symmetry. If the line of symmetry treated as boundary then the number of nodes would be greatly reduced. The finite differences equation corresponding to this line is[6]:

$$
V_{i j}=\frac{1}{6}\left[4 V_{(1, j)}+V_{(0, j-1)}+V_{(0, j+1)}\right]
$$

Along boundary line $(\mathrm{C})$, for no current flow across it, then the finite differences equation corresponding to this line is:

$$
V_{i j}=\frac{1}{4}\left[\left(1-\frac{1}{2 i}\right) V_{(i-1, j)}+\left(1+\frac{1}{2 i}\right) V_{(i+1, j)}+V_{(i, j-1)}+V_{(i, j+1)}\right]
$$

This equation is also used in the boundary (D), with changing $V_{(i, j+1)}$ to the sink voltage (SNKV)[7].

$$
S N K V=P H I O-(S B V D+A N V D)
$$

where $P H I O$ is a voltage at the stub, $S B V D$ is stub-anode voltage drop, and $A N V D$ is the voltage drop across the carbon anode.

\section{Contact Resistance}

The concept of the contact resistance was used to define the interfacial boundary conditions. It is derived from ohm's law, which is valid across an interface, since it is assumed that the current flow across it undergoes no divergence. The ohm's law is given as:

$$
V=\frac{I}{A}(\rho l)
$$

where $I$ is the current through the interface, $\rho$ is the specific resistance of the interface material, $l$ is the thickness of the interface, and $A$ is the area of the interface. In this study the value of $0.6186 \times 10^{-2} \Omega . \mathrm{cm}^{2}$ was used for contact resistance between carbon and cast iron [8].

\section{Calculation of AnOde Life Time}

To product one kilogram of $\mathrm{Mg}$, it needs the energy of $\left(30.6 \times 10^{6}\right) \mathrm{J}$. The calculation of the amount of carbon consumption to produce one kilogram of pure $\mathrm{Mg}$ according to reaction [9]:

$$
\begin{aligned}
& \mathrm{MgO}+\mathrm{Cl}_{2}+\mathrm{C} \longrightarrow \mathrm{MgCl}_{2}+\mathrm{CO} \\
& \mathrm{MgCl}_{2}+\text { electric energy } \longrightarrow \mathrm{Mg}+\mathrm{Cl}_{2}
\end{aligned}
$$

It is clear from the above equation reaction, a mole of $\mathrm{Mg}$ as product exile one mole of carbon anode. Since atomic weight of $\mathrm{Mg}$ equal to (24.305), and atomic weight carbon is (12); the mass of carbon consumption $m_{C}$ to produce one kilogram of pure $\mathrm{Mg}$, is equal to $(12 / 24.305=0.4937) \mathrm{Kg}$.

So the energy required to consume $E_{c}$ one kilogram of carbon is:

$$
E_{c}=\frac{30.6}{0.4937}=61.98 \mathrm{~J} / \mathrm{Kg}
$$

By using the power of the cell, the average life time of each anode is determined using the following relation:

$$
t_{c}=\frac{E_{c} \rho_{c}\left(\pi R_{c}^{2}\right) h_{a}}{I V_{S}}
$$

where $\rho_{c}$ is the carbon density of the anode, $h_{a}$ is the active length of the anode, $R_{c}$ is carbon radius, and $V_{s}$ is the sink voltage at the electrolyte interface.

\section{Finite DUfFERENCE AND COMPUTER PROGRAM}

The calculation and analysis of potential represent an important stage in the design of anode in the Dow cell. The familiar analytic methods (Separation of variables, the methods of images, etc.) are suitable for an extremely restricted group of problem [10]. Therefore, numerical methods, such as finite element and finite difference must be carry out for calculating voltage distribution in carbon anode cell [11]. The advantage of the finite element method is its simplicity in treating problems with inhomogeneous media and with very complicated boundary [12], but the problem concerned is a homogeneous and very complicated boundary, therefore, the finite difference method is used. In this study, the Laplace's equation takes the familiar central difference form as:

$$
V_{i j}=\frac{1}{4}\left[V_{(i-1, j)}+V_{(i+1, j)}+V_{(i, j-1)}+V_{(i, j+1)}\right]
$$

The numerical interactive successive over relaxation method [13] was used to solve this equation with suitable boundary conditions. The advantage of this method in this application gives a simple and compact program textually and uses relatively little computer store.

The stub region is assumed to be at uniform potential. To run the program it is first necessary to specify the appropriate anode geometry, carbon resistivity, current, and contact resistance values. It is also necessary to specify an arbitrary starting potential drop, line boundary conditions, and re-entrant corner point. A distribution voltage is then calculated by solving the system of equations. Many efforts are devoted to manipulating and finding the optimum successive over relaxation factor during solution of the equation, iterations are carried out to a residual error.

\section{CONVERGENCE RAT FACTOR}

Fig. 2 shows the effect of various convergence rate factor on the iteration number (with supposing the maximum value of iteration was 500). This figure clearly indicates the most suitable value for obtaining the minimum iteration number was (1.93), thus its value is fixed in the program.

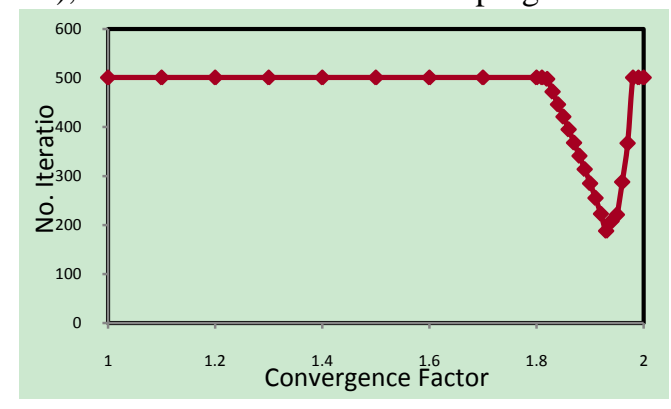

Fig. 2. The number of iterations of the program required for convergence of the convergence of the convergence factor.

\section{EFFECT OF RESISTIVITY CONTACT OF ANODE AND STUB}

Two main simulation have been carried out, the first was based on the assumption the good electrical contact existed down the vertical side of the cavity (region E in Fig. 1) but poor electrical contact was assumed to exist in the region $\mathrm{G}$. in the second existed we assume an equal good contact in two 
regions. The results of first simulation are shown in Fig. 3(a); iso-potential line have been drawn in $(20 \mathrm{mV})$ steps and current at any point normal to these lines, it can be seen from the high concentration of lines at the side of the cavity that current predominately enters the block in this region, it then diverges to give a fairly uniform distribution at the bottom of the anode. The mean anode voltage drop was found to be $(363 \mathrm{mV})$ the mean baseline voltage of $(3.637 \mathrm{~V})$ represented the voltage drop through the electrolyte.

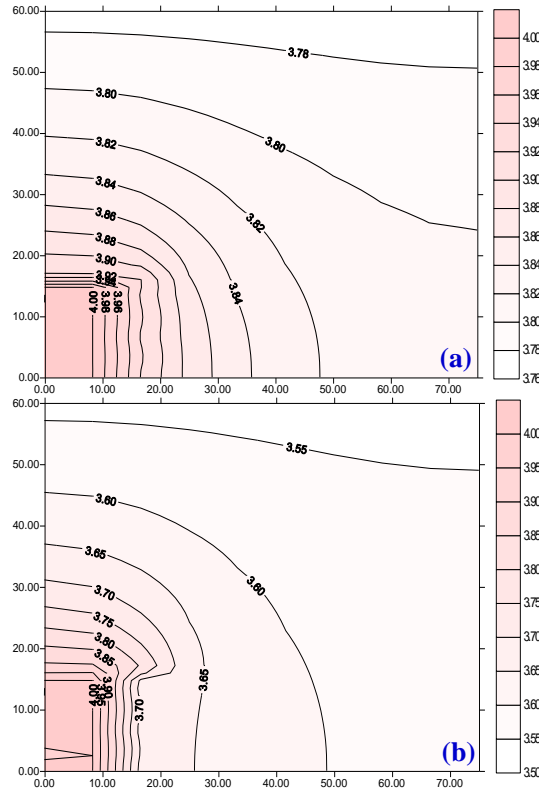

Fig. 3. The potential distribution as a function of $(r, z)$ for: (a) poor electrical contact. (b) good electrical contact, in the region G.

Results from the second simulation are shown in Fig. 3(b). The benefit of improving the electrical contacts all around the stub can be seen by much more uniform flow into the anode. The voltage drop across the anode in this case was (257 $\mathrm{mV}$ ), representing a save of $(106 \mathrm{mV})$ compared with first simulation. This self-saving dies not appear large, but when it is realized that $(7200 \mathrm{~A})$ is passing through each of the anodes then the total power saving is appreciable (763.2 Watt).

\section{The First CONFIGURATION OF ANODE}

The anode configuration is chosen as a cylindrical shape with radius $(30 \mathrm{~cm})$, and height $(60 \mathrm{~cm})$, while the stub is made of iron in the form of cylindrical shape with radius (12 $\mathrm{cm})$, and height $(15 \mathrm{~cm})$.

The symmetry is taken into consideration when solving equations by finite difference to reduce the computational work. Due to symmetry of anode around $\varphi$-direction, only one half of it is considered. Square mesh $(20,40)$ system was imposed on the anode, with intervals $(1.5 \mathrm{~cm})$ in both $\mathrm{r}$ and $\mathrm{z}$ directions. The voltage at the stub was assumed to be $(4 \mathrm{~V})$ with current $(7200 \mathrm{~A})$.

The contour potential distribution as a function of $r$ and $z$ with accuracy of $\left(10^{-4}\right)$, are shown in Fig. 4. It is clear from figure that the voltage distribution around the stub is irregular, therefore, it caused to a large amount of electric power to dissipate.

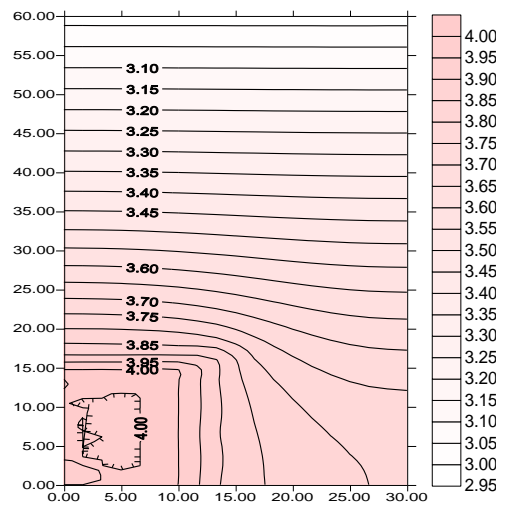

Fig. 4. The potential distribution as a function of $(r, z)$.

The parameters of this configuration are presented in Table(1), in which the drop voltage and the power dissipation are not small, while the life time of the cell is very short which are contracted with the aim of this work. Thus incentive considered the following design in order to obtain the optimum configuration satisfied with increasing the life time and decreasing the power dissipation.

TABLE I: The MAIN PARAMETER OF THE ANODE With RADIUS (30 CM), AND HEIGHT (60 CM).

\begin{tabular}{clll}
\hline No. & Parameter of the Anode & Value & Unite \\
\hline 1 & Activation height & 27 & $\mathrm{~cm}$ \\
2 & Carbon anode voltage drop & 0.94515 & Volte \\
3 & Total voltage drop & 1.05076 & Volte \\
4 & Power dissipation & 6805.1 & Joule \\
5 & Life time & 5.7255 & Days \\
6 & Magnesium produce & 83670 & $\mathrm{Kg}$ \\
\hline
\end{tabular}

\section{The Effect of Anode Cross Section}

To determine the optimum dimensions of the Dow cell, the effects of various cross section of Carbon anode are investigated; All the configuration with the same physical properties (resistivity of the carbon $=0.6186 \times 10^{-2} \Omega . \mathrm{cm}$, voltage supply on the stub $=4 \mathrm{~V}$, total anode current $=$ $7200 \mathrm{~A}$, and anode stub contact resistance $=0.2322 \times$ $10^{-1} \Omega . \mathrm{cm}$ ). Nine different anode base areas with equivalent highs are examined. The potential distributions for different anode cross sections are shown in Fig. 5. It is observed that the voltage distributions are regular for both dimensions, and the drop voltage decreases with increasing base area of the anode. This refers to decrease of the current density within the carbon anode by increasing base area.

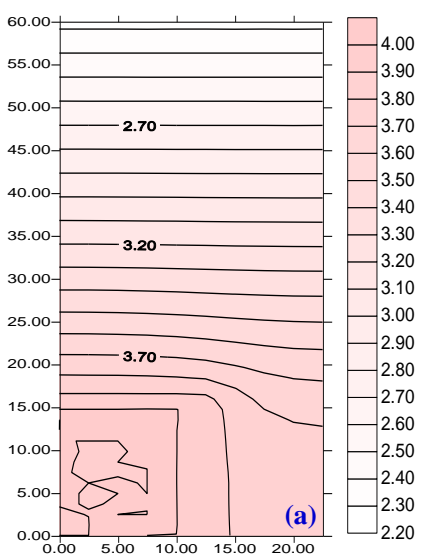




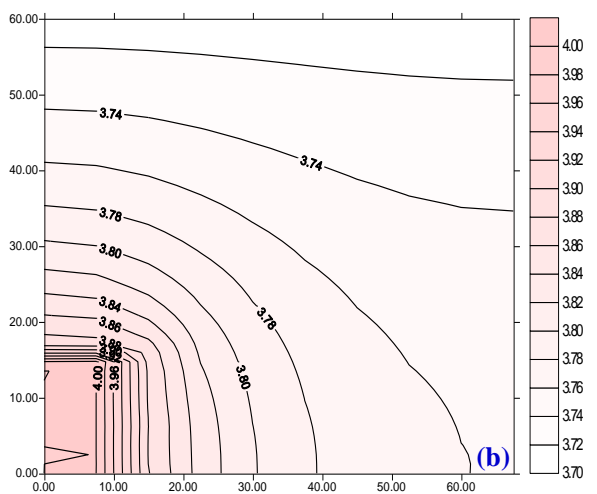

Fig. 5. The potential distribution as a function of $(r, z)$, for different anode radius (a) $r=22.5 \mathrm{~cm}$, (b) $r=67.5 \mathrm{~cm}$.

The effect of anode radius on the life time and the power dissipation of the cell, was shown in Fig. 6. It was clear that the increase of anode radius cause increases of life time of the cell, and decreases the power dissipation. So the highs anode radius has the best performance.

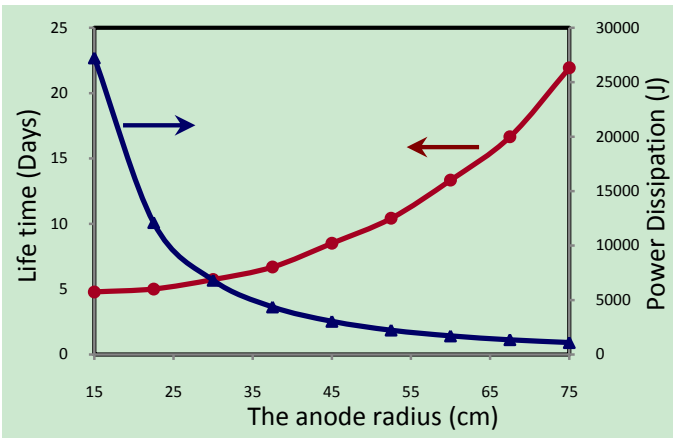

Fig. 6. Life time and power dissipation of the cell as a function of anodes radius.

\section{The Effect of Stub Cross Section}

It seems from the previous section that the optimum configuration is the greatest anode radius, therefore; the anode dimension is fixed with radius $(75 \mathrm{~cm})$. The different stub radius were taking in the range $(7.5-18) \mathrm{cm}$, with the same physical parameters as the previous section. The potential distributions for different stub cross sections are shown in Fig. 7. It can be noted that the drop slightly effected with increasing base area of the stub.

The life time and the power dissipation of the cell as a function of stub radius are shown in the Fig. 8. The increase of stub radius leads to decreases of life time of the cell, while the power dissipation remains constant at the minimum.

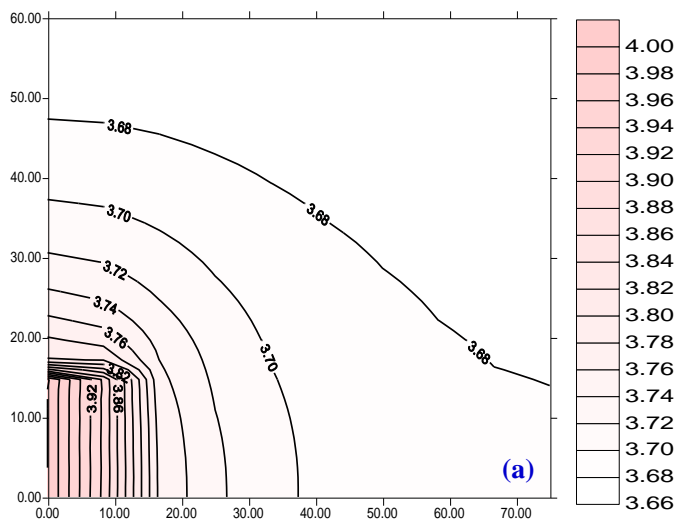

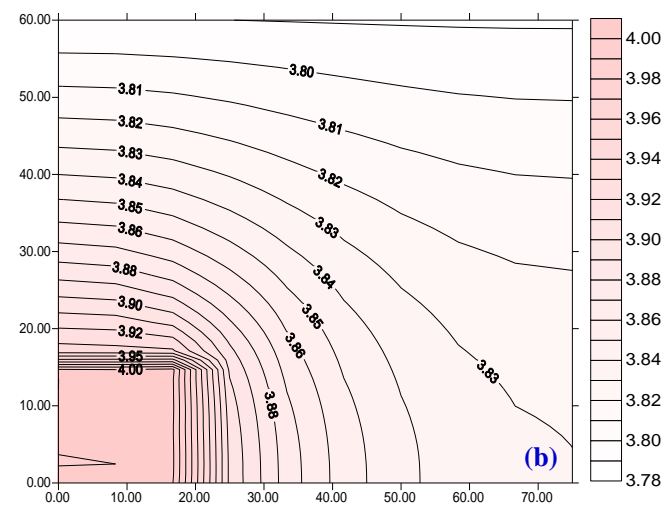

Fig. 7. The potential distribution as a function of $(r, z)$, for different stub radius $(a) r=7.5 \mathrm{~cm},(b) r=18 \mathrm{~cm}$.

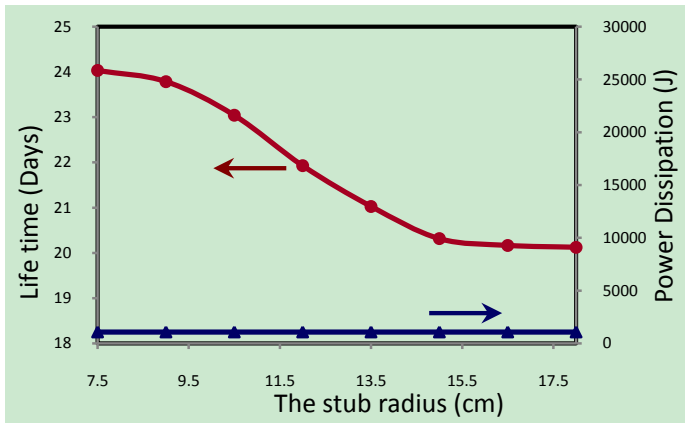

Fig. 8. Life time and power dissipation of the cell as a function of stub radius

\section{CONCLUSION}

The finite difference method was used it investigate the voltage distribution within the anode Dow cell, and some important parameter like life time, power dissipation, and magnesium product are considered. The simulations have indicated that appreciable power saving could be achieved by improving the electrical contact at the base of the stub cavity. The increase of carbon anode cross section shows an increase of life time and decrease of power dissipation. While the increases of stub cross section caused decrease in life time of the cell with fixed value of power dissipation.

\section{REFERENCES}

[1] D. Kasherman and M. Skyllas-Kazacos, "Effects of anode-cathode distance on the cell potential and electrical bath resistivity in an aluminium electrolysis cell with a sloping TiB2 composite cathode," Journal of Applied Electrochemistry vol. 18, no. 6, pp. 863-868, 1988.

[2] W.-S. Chen, "Optimal design of high voltage insulators using genetic algorithm and charge simulation method," PhD Thesis, Chung Yuan Christian University, Taiwan, 2008.

[3] S. K. Banerjee and J. W. Evans, "Measurements of magnetic fields and electromagnetically driven melt flow in a physical model of a hall-heroult cell," Metallurgical and Materials Transactions vol. 21B, no. 1, pp. 59-69, 1990.

[4] A. D. Sneyd, "Interfacial instabilities in aluminium reduction cells," Journal of Fluid Mechanics, vol. 236, pp. 111- 126, 1992.

[5] D. Richard, M. Fafard, R. Lacroix, P. Clery, and Y. Maltais, "Aluminum reduction cell anode stub hole design using weakly coupled thermo-electro-mechanical finite element models," Finite Elements in Analysis and Design, vol. 37, no. 4, pp. 287-304, 2001.

[6] B. F. Bradley, D. W. Macmillan, and S. P. Castell, "Voltage distributions in an aluminium smelter prebaked anode," Applied Mathematical Modeling, vol. 1, no. 6, pp. 339-343, 1977.

[7] M. G. Say, "Field analysis: experimental and computational method," Edited by D. Vitkovitch, Van Nostraind 1966.

[8] D. Richard, M. Fafard, R. Lacroix, P. Clery, and Y. Maltais, "Carbon to cast iron electrical contact resistance constitutive model for finite element analysis," Journal of Material Processing Technology, vol. 132, no. 1-3, pp. 119-131, 2003. 
[9] W. H. Hayt and J. A. Buck, "Engineering electromagnetic," McGraw-Hill, sixth edition, 2001.

[10] H. H. Daumling and H. Singer, "Investigation on field optimization of insulator geometries," IEEE Transaction on Power Delivery, vol. 4, no. 1 , pp. 787-793, 1989.

[11] O. W. Andersen, "Two stage solution of three dimensional electrostatic fields by finite differences and finite elements," IEEE Transactions on Power Apparatus and Systems, PAS-vol. 100, no. 8, pp. 3714-3721, 1981.

[12] M. V. K. Chari and P. P. Silvester, "Finite elements in electrical and magnetic field problem," John Wiley and Sons Inc, 1980.

[13] J. D. Hoffman, "Numerical methods for engineers and scientists," Marcel Dekker Inc., Second Edition 2001.

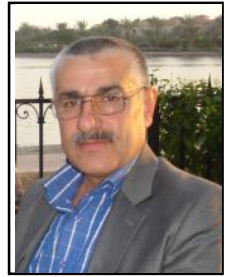

Othman S. Hamad was born in Erbil, Kurdistan Region-Iraq, on July 1963, He received the M.Sc. degree in Material Science from Baghdad University, in 2000; and $\mathrm{PhD}$ degree in Electromagnetic theory from Baghdad University, in 2007. Now he is a lecturer in physics department, College of ScienceEducation, University of Salahaddin-Erbil, Iraq. His principle research interests have been in the fields of
Anod design, Girotron, and finite different method. He is a member of Iraqi Physics and Mathematics Society, Kurdistan Physicists' Syndicate.

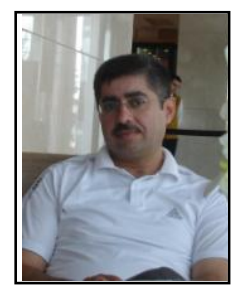

Omed Gh. Abdullah was born in Sulaimani, Kurdistan Region-Iraq, on July 1969, He received the M.Sc. degree in Material Science from Baghdad University, in 1999; and $\mathrm{PhD}$ degree in Material Science from Baghdad University, in 2006. He is worked as a lecturer in the Salahaddin UniversityErbil, and University of Sulaimani. Now he is assistant professor in physics department, School of Science, University of Sulaimani, Kurdistan Region-Iraq. His principle research interests have been in the fields of solid state physics, material science, and powder technology. His interests include fabrication and characterization of thin films, physical properties of composite material, and simulation of powder compaction. He is author or coauthor of 21 publications in refereed journals. He is a member of IACSIT, Iraqi Physics and Mathematics Society, Kurdistan Physicists' Syndicate, and Kurdistan Chemists \& Physists Society. 